\title{
Cryptococcus Meningitis, in the Patients with Human immunodeficiency Virus Infection
}

\author{
${ }^{1,}$ Murtaza Mustafa , ${ }^{2,}$ FA.Mohamad Salih , MTH. Parash , ${ }^{3,}$ CH.Sadiah Shimmi, \\ ${ }^{4}$ MD.Shamsur Rahman \\ ${ }^{1,2,3,4,}$ Faculty of Medicine and Health Sciences, University Malaysia Sabah,Kota Kinabalu, \\ Sabah Malaysia.
}

\begin{abstract}
AIDS have given more importance to cryptococcosis, which was discovered a century ago.Cryptococcus neoformans is the most frequent cause of meningitis in HIV infected patients. Cryptococcus can infect any organ in the human body, the frequent sites are lung,CNS, skin,eye and prostrate The impact of capsular polysaccharide,melanin and ability to grow at higher temperature, in host immunity can be profound at pathophysiologic levels. Cryptococcal meningitis presentation in AIDS patients may be very subtle, with headache,fever, and lethargy. Meningeal signs occurs in some patients, photophobia and cranial nerve palsies are often absent, most patients have CD4 T-cell lower than 50 cells/ $\mu$ l.African patients have higher rate of neurologic compromise. Strong cellular immune response producing granulomatous inflammation is essential for containment of infection. Pathophysiology of cryptococcosis is similar to reactivation of tuberculosis, and histoplasmosis.Diagnostic methods include, direct microscopic examination by India ink mixing of biologic fluids, detection of polysaccharide antigen and a positive blood culture. Treatment of choice in AIDS patients with cryptococcal meningitis with amphotericin B with flucocytosine or fluconazole for 4 to 6 weeks Monotherapy cannot be recommended for the treatment of this infection.
\end{abstract}

KEYWORDS: Cryptococcous neoformans,Meningitis,Acquired immunodeficiency syndrome(AIDS).

\section{INTRODUCTION}

Cryptococcus was discovered by Sanefelice in 1894, from peach juice in Italy[1].Within a year, Busse and Buschke independently reported the first human case of cryptococcosis in a young woman[2].In 1914, Verse described a human case of cryptococcal meningitis, and in 1916,Stoddard and Cutler gave a complete description of CNS pathology for this infection including in their report that yeast forms have had surrounding areas of clearing within the tissue[3].In1976,Kwon-Chung discovered and characterized the sexual stage of Cryptococcus and the teleomorphs were named Filobasidella neiformans and Filbasidella bacillospora[4].In 2002, it was proposed that Cryptococcus have two varities, and later the genome sequence was released, as we enter the genome age of fungal pathogens [5,6].There is no established skin test for routine clinical use in patients with cryptococcosis, and this reduces the ability to assess the magnitude of infection. However, most adults possess antibody to C.neoformans antigens;in New York City, most children acquire antibodies to cryptococcal antigens before the age of 10 years[7]. The most underlying conditions worldwide include the acquired immunodeficiency syndrome(AIDS),prolonged treatment with corticosteroids, organtransplantation,advanced malignancy,diabetes, and sarcoidosis.Cryptococcosis may identify idiopathic CD4 lymphocytopenia[8].

The best estimates for rates of cryptococcosis in the United States in pre-AIDS era predicted an overall incidence of 0.8 case/million persons per year. In 1992,during the peak of the AIDS epidemic in the United States, the rate reached almost 5 cases of cryptococcasis $/ 100,000$ persons per year in large cities[9]Human-tohuman transmission of cryptococcosis has not been reported, except in cases of contaminated transplant tissues[10].However, in one case C.neoformans isolated from the cage of pet cockatoo was molecularly linked the strain that caused infection in a transplant recipient who was exposed to the cage Also several cryptpcoccal cases have been linked to intense bird exposure[11,12].Cryptococcal meningitis presents differently in AIDS and non-AIDS patients. Presentation in AIDs patients may be very subtle, with headache,fever,and lethargy. Meningeal signs occur in some patients. Photophobia and cranial nerve palsies often are absent. In African continent AIDS patients with cryptococcal meningitis have higher rates of neurologic compromise [13]. The drugs of choice in AIDS patients with a first episode of AIDS-associated cryptococcal meningitis to amphotericin $\mathrm{B}(0.7 \mathrm{mg} / \mathrm{kg} /$ day $)$ with or without 5 -flucytocine $(5-\mathrm{FC}),(100 \mathrm{mg} / \mathrm{kg} /$ day) for two weeks followed by 8 weeks of treatment with itraconazole $(400 \mathrm{mg} / \mathrm{day})$ or fluconazole(400mg/day)[14].In non-AIDS patients. 
Combination therapy with amphotericin B plus 5-flucytosine for 4 to 6 weeks [15].The paper reviews the epidemiology pathogenesis, clinical manifestation, and therapy of Cryptococcus meningitis in AIDS patients.

\section{CRYPTOCOCCUS AS A PATHOGEN}

The encapsulated yeast C.neoformans has been studied extensively for more than 50 years. In the past decade genetic and molecular biologic research, in concert with well- established and robust animal models, has rapidly increased our understanding of its pathobiology. Progress in cryptococcal molecular biology has led to the use of karyotypes,repetive elements,transposons and sequencing to identify yeast strains through a variety of analytical techniques, including restriction fragment length polymorphism(RFLP,RAPD,PCR finger- prints and MLST.Recently the entire genomes of several strains of C.neoformans and.C gattii have been sequenced[16].Furthermore, differential display PCR,cDNA subtractiontechniques, serial analysis of gene expression(SAGE), and microarray analysis have been used to document and understand C.neoformanstranscription profiles [17].

Capsule or slime layer : The most distinguished feature of C.neoformans is a polysaccharide capsule containing a unbranched chain of $\alpha$-I,3-linked mannose units substituted with xylosyl and $\beta$-glucuronyl groups. The serotype specificity appears to be determined by structural differences in the glucuronoxylomannan(GMX) related to the number of xylose residues and the degree of 0-acetylation of hydroxyl groups. The capsular polysaccharide has high negative cell surface charge and is attached to the cell wall by $\alpha$-A,3-glucan residues.However,it is easily released into the immediate growth media or tissue. Capsular thickness, which varies among isolates, is regulated by several environmental cues, including both ambient Pco2,serum, and low iron concentrations, which increase capsular size in many strains. These environmental signals appear to augment the yeast's ability to produce disease and may help explain why the capsule may be small in in vitro cultures but is much larger in when observed in the host. Mutant Cryptococci that are made to be hypo capsular or acapsular are dramatically less virulent than the parental strains in animal models[18].The impact of the capsular polysaccharide on host immunity can be profound at many pathophysiologic levels[19]that include:(1)an antiphagocytosis barrier(2)depletes complement(3)produces antibody unresponsiveness(4)deregulates cytokine secretion(5)interferes with antigen presentation(6)produces brain edema(7)creates selection and tumor necrosis factor receptor loss(8)allows a highly negative charge around yeast cells(9)extrudes itself into the intracellular environment with the potential for local toxicity on cellular organelles(10)enhances HIV replication.

Production of Melanin : The production of melanin is observed in many fungi including some pathogenic fungi [20].C.neofomans possesses laccase,anenzyme that catalyze the conversion of diphenolic compounds such as I-DOPA,norepinephrine, and other related aromatic compounds to quinones. which rapidly autopolymerize to form melanin that is bound to the inner aspect of the yeast's cytoplasmic membrane.The production of this pigment can help identify the yeast in the laboratory, but it is also a major virulent factor for the yeast [21]. One proposed mechanism whereby melanin may protect the yeast is through its ability to act as antioxidant, and it has shown that yeast cells without the ability to form melanin are more susceptible to oxidation stress. Other mechanisms whereby melanin protects the yeast from host damage include (a) cell wall support or integrity (b) alteration in cell wall charge(c) interference with T-cell response (d) abrogation of antibody-mediated phagocytosis (e) protection from temperature changes. It remains unclear whether the catecholamine-rich CNS, with its excellent substrates for melanin formation, provides some tissue tropism or rich environment that enhances the yeast ability to produce disease. However, it has clearly been shown that melanin is formed in yeast cells within the brain cells [22].

Ability to grow at high temperature: The basic trait of all pathogenic fungi is their ability to grow well a mammalian body temperature. For example C.neoformans and C.gattii are the only cryptococcal species to grow at $37^{\circ} \mathrm{C}$, and when mutants are made that cannot grow well at this temperature, they are avirulent ,even when they can make capsule and produce capsules and produce melanin. There appears to be some evolutionary drift in high -temperature growth in that isolates of C.gattii and serotype $\mathrm{D}$ (var.neoformans)generally appear to be more sensitive to growth inhibition and killing at high temperature than serotype $\mathrm{A}(\mathrm{Var}$.grubii)and less heat tolerant isolates quickly lose viability at temperature of $40^{\circ} \mathrm{C}$ and above[23].There has been progress in understanding the genetic controls for high-temperature growth in C.neoformans.First two signaling pathways(calcineurin and RAS) have been associated with the yeast's ability to grow at mammalian body temperature, and there are these linked to its virulence composite[24].Detailed research has focused on three classic virulence factors of C.neoformanse.capsule, melanin and growth at $37^{\circ} \mathrm{C}$,but this complex pathogen has many other tools to produce disease. First individual clinical or environmental strains vary in their ability to produce in animal models, despite possessing all known virulence factors. Strains can also change their 
virulence potential by passage through animals. Although many strains are clonal, there have been recombination events in nature to produce $\mathrm{A} / \mathrm{D}$ hybrids and progeny that are more fit or virulent than the parental strains [25].

\section{CRYPTOCOCCUS INFECTION AND HOST RESPONSES}

Serologic and skin hypersensitivity studies frequently identify cryptococcal infections and yet the incidence of cryptococcosis is low, it has been concluded that host immunity in humans is generally effective after initial exposure to this yeast[26].In fact, the vast majority of cryptococcal infections are diagnosed in patients with compromised cell-mediated immunity. Furthermore, there is general agreement among clinicians that a strong cellular immune response producing granulomatous inflammation is essential for containment of infection [26].Several immune cells populations, such as natural killer cells, and certain types of lymphocytes have been shown to possess direct anti cryptococcal effects. Human lymphocytes(CD4,CD8) inhibit the growth of C.neformans by direct contact [27].A primary effector cell against C.neofomans is the macrophage, which produces anticryptococcal activity when activated [28].It is clear from natural history studies in patients with AIDS that risk of infection dramatically increases as totalCD4 lymphocyte count drop below 50 to 100 cells $/ \mu \mathrm{L}$ of blood In these patients, the paucity of inflammatory cells at the site of infection, such as the subarchnoid space is impressive[29]. There is substantial evidence that the humoral immunity arm can contribute to an effective immune response. Several groups have shown that monoclonal antibody strategies directed against the polysaccharide capsule can reduce the burden of yeasts and improve the survival in model animals[30].A pilot study in humans has been performed to determine the safety and dynamics of murine monoclonal antibody for treatment of cryptococoosis [31].

\section{PATHOPHYSIOLOGY}

The pathogenesis of cryptococcosis is determined by three broad factors:(1)the status of the host defenses(2) the virulence of the strain C.neoformans(3)the size of the inoculum. The relative importance of each factor as determinant of clinical disease remain uncertain, but it is clear that complexities of these interactions together produce the ultimate presentation[32].A reasonable scenario for the pathophysiology of cryptococcosis is that the susceptible host comes into contact with Cryptococci from the enviromment through inhalation of infectious propagules, In the alveoli,the yeasts contact the alveolar macrophages, which recruit other inflammatory cells through cytokines,and a proper Th I response and granulomatous inflammation is elicited.The infection can take three pathways[32].(1) In an immunosuppressed host, the yeast continues to proliferate and disseminate, causing clinical disease.(2)The effective immune response completely eliminates the yeast from the host(3)The yeast produce a small lung-lymph node and remain dormant in tissues but not dead. The third scenario may be common occurrence. Baker and colleagues, in elegant postmortem studies of asymptomatic individuals, showed the existence of pulmonary foci and hilar nodes containing yeasts in individuals with no antecedent complains[33]. The yeast remain dormant and the host is clinically asymptomatic until loss of local immunity occurs through, for example, corticosteroids use or progression of an HIV infections. Then , the yeasts begin to replicate in the pulmonary lymph node complex and eventually disseminate into organs outside lung. This pathophysiology is similar to the scenario proposed for reactivation of tuberculosis and histoplasmosis.Studies in France have given epidemiologic support for this concept of reactivation in African ex-patriots who lived in Europe for many years prior to their development of cryptococcosis, the infecting strain possessed a genotype consistent with strains from an African strain[34].

\section{CLINICAL PRESENTATION}

C.neoformans found to infect any organ of the human body[19].The common sites of for infection with this encapsulated yeast, the lung and CNS, were emphasized in review of cryptococcosis in HIV-negative patients[35].In this cohort,109(36\%) were diagnosed with only pulmonary involvement and 157(51\%) presented with initial evidence of CNS disease. Three other sites of infection are skin, prostrate and eye have clinical features worthy of mention [36]Cryptococcosis demonstrates a few differences, depending on whether the patient has or does not have an underlying HIV infection.HIV-infected patients present with more CNS and extrapulmonary infections, higher rates of positive India ink examinations. Higher polysaccharide antigen titers, more frequent positive blood cultures, and fewer cerebrospinal fluid (CSF) inflammatory cells. These clinical distinctions are primarily a function of the severity of immuno-nonsuppression and the resulting high burden of yeast. They most likely do not reflect a specific interaction between HIV and C.neoformans growth [37].

Central nervous system infection :In CNS infection most patients with cryptococcosis present with signs and symptoms of subacute meningitis or meningoencephalitis,such as headache,fever,cranial nerve palsies, lethargy,coma or memory loss over several weeks[35].Symptoms may not be typical, and patients may present with acute(several days) symptoms of severe headaches, with intermittent headaches, or even no headache but 
with altered mental status[32].HIV-infected patients with cryptococcal meningitis exhibit few differences at presentation from those without HIV.However,several clinical aspects may be more prominent in patients with AIDS[36] that include:(1)the burden of yeast is generally higher, and this may be reflected in higher polysaccharide antigen titers, slower conversion of CSF to sterilization during treatment, and a tendency toward a higher incidence of increased intracranial pressure.(2)there is greater likelihood of finding the yeast in extracranial locations during the initial workup.(3)the possibility is greater that a second CNS event may occur, such as infection with Toxoplasma gondii or development of a lymphoma.(4)the use of HARRT in AIDS patients has created a new immune reconstitution syndrome in cryptococcal infection[38].It appears in two forms, unmasking and paradoxical. In the unmasking form, after starting HARRT, some patients develop acute symptoms of cryptococcal meningitis or pain and swelling in peripheral,hilar or mediastinal lymph nodes. In the paradoxical form, new symptoms may also occur during otherwise successful treatment of cryptococcal meningitis in the first weeks after HARRT is introduced. It appears to correlate with significant drop in HIV load, but there may be only a modest rise in the number of CD4 cells [39].

It is hypothesized that as immunity improves with HARRT,silent or latent cryptococcal infections are made clinically apparent as inflammation is mobilized to interact with the yeast or polysaccharide antigen. During treatment for cryptococcal meningitis this immune reconstitution syndrome may be marked by increasing headaches, new neurologic signs, appearance of more inflammatory cells in the CSF,and possibly more intracranial pressure [40].Distinction between cultures from the CSF and lymph node aspirates are generally negative in immune reconstitution syndromes, even though cryptococci may be present on a smear[32].There are limited data that relate the severity of the meningitis to the particular infecting strain and, in most cases, the hos defense responses determine the clinical manifestations.However,some clinical presentations may depend on the particular infecting strain. For example, in areas of the world that have infections with both C.neoformans serotypes $\mathrm{A}, \mathrm{D}$, and $\mathrm{AD}$ (var neoformans or grubii) and C.gattii,cerebral cryptococcomas and hydrocephalus with or without large pulmonary mass lesions in immunocompetent hosts were more commonly with C.gattiiinfections[41].Another example of strain affecting disease production is from the Vancouver outbreak, wherein it was found that recombinant strain was created in nature that was more virulent in animal models than the parental strain and had taken over the environmental and clinical isolates in this outbreak[25].

\section{DIAGNOSTIC METHODS}

Direct microscopic examination.Dark field microscopy or by India ink mixing of biologic fluids to identify the 5-to $10 \mu$-diameter encapsulated yeasts remains a rapid and effective method for diagnosing cryptococcal meningitis. Approximately 50\% of non AIDs patients with cryptococcal meningitis and over $80 \%$ of patients with AIDS have positive India ink examination of CSF.The polysaccharide capsule can be identified with stains such as mucicarmine and Alcian blue and its ability to produce melanin allows it to be stained with FontanaMasson stain.Gomori's methenamine silver(GMS) fungal stain identifies the narrow-based budding yeast in tissue, and Gram stain usually reveals a poorly stained gram-positive yeast. Both biopsies and cytologies can be helpful in the diagnosis of cryptococcosis[32].

Serologic tests.The serologic tests for the detection of cryptococcocal polysaccharide antigen in serum and CSF are extremely accurate for the diagnosis of invasive disease. Both latex agglutination and enzyme immunoassay tests are more than $90 \%$ sensitive and specific $[42,43]$. With the proper treatment of specimens(boiling and pronasem2-mercaptoethnol treatment),false positive tests are not common when CSF titers are 1:4 or higher[44].

Culture methods.C.neoformans can grow on most bacterial and fungal media. Both automated and lysis centrifugal methods are effective in detecting cryptococcemia,and the finding the positive blood cultures has been more common during the AIDS epidemic.Most C.neoformans isolates from untreated patients can be detected in culture 3 to 7 days after the specimen collected and placed into or on culture media. Isolates can be identified by biochemical reactions[45], or DNA based methods[46]. Other methods to presumptively identify the yeast are to perform a rapid urease test or to inoculate the yeast onto Staib's birdseed,or DOPA,or Caffeic acid media(in which colonies will produce melanin and turn brown to black[47].

\section{TREATMENT}

Amphotericin B remain the cornerstone of therapy for cryptococcal meningitis and from early studies when it was used alone to its use in combinations, it has performed reasonably well for this infection with successes in the non-AIDS era of between $60 \%$ to $75 \%[48,49]$. With polyene therapy,two issues have been noted.First,studies have suggested that higher daily doses of amphotericin B might be more effective in sterilization of the CSF.A standard induction dose for amphotericin B deoxycholate has now been established at $0.7 \mathrm{mg} / \mathrm{kg} /$ day .Second,liposomal amphotericin B(AmBisome) and amphotericin B lipid complex(ABLC) at 3 to 
$6 \mathrm{mg} / \mathrm{kg} / \mathrm{day}$ have had treatment success similar to that of amphotericin B deoxycholate, with reduced toxicity[50,51].Flucytocine has been used alone in treatment of cryptococcal meningitis, but frequent development of direct drug resistance on monotherapy means that it cannot be recommended as a single agent for the treatment of this infection. It is primarily used in combination therapy with amphotericin $B[52,49]$.

\section{CONCLUSION}

Cryptococcus meningitis in AIDS patients is an opportunistic infection.Prevention and treatment of this infection in HIV patients is significantly reducing the mortality among HIV patients.

\section{REFERENCES}

[1] Sanfelice F.[Contributo alla morfolgia e biologia dei blastomiceti che si sviluppano nei succhi di alcuni fruiti]Ann dIgiene.1894;4:463-65.

[2] Buschke A.[Ueber eine durch coccidian hervogerufene krankheir des menschen]Dtsch Med Wochenschr.1895;21:14.

[3] Verse M.[Ober einen Fall von generallisierrter Blastomykose beim Menschen]VerDisch Path Ges.1914;17:275-78.

[4] Kwon -Chung KJ.Morphogenesis of Filobasidella neoformans the sexual state of Cryptococcus neoformans.Mycologia.1976;68:821-833.

[5] Kwon-Chung KJ,Boekhour T,Fell JW,et al.Proposal to conserve the name Cryptococcus gattii against C.hondrianus and C.bacillisporus(BlastomycotaHymenomycetes Tremellomycetidae)Taxon.2002;51:804-806.

[6] Van Duin D,Casadevall A,Nosanchuk JD.Melanization of Cryptococcus neoformans and Histoplasma capsulatum reduce their susceptibilities to amphotericin B and caspofugin .Antimicrob Agent Chemother.2002;46:3394-3400.

[7] Chen L-C,Goldman DI,Doering TI.Antibody response to Cryptococcus neoformans in rodents and humans.Infect Immun.1999;67:2218-24.

[8] Zonios DI,Falloon J,Huang CY,et al.Cryptococcus and idiopathic CD4 lymphocytopenia.Medicine(Baltimore).2007;86:78-92.

[9] Hajiman A,Conn LA,Stephens DS.Cryptococcus:Population-based multistate active surveillance and risk factors in human immunodeficiency virus infected persons.Cryptococcal active Surveillance Group Infect Dis.1999;179:449-54.

[10] Beyt BE,Waltman SR.Cryptococcal endophthalmitis after corneal transplantation .N.Engl J Med.1978;298:825-26

[11] Nonsanchuk JD,Shoham S,Fries BC,et al.Evidence for zoonotic transmission of Cryptococcus neoformans from a pet Cockatoo to an immunocompromised patient.Am Intern Med.2000;132:205-208.

[12] Fessel WJ.Cryptococcal meningitis after the unusual exposure to birds.N Engl JMed.1993;328:1354-55.

[13] Mossa MYS,Coovadia YM.Cryptococcal meningitis in Durban, South Africa: a comparison of clinical features, laboratory findings, and outcome for human immunodeficiency virus(HIV)-positive and HIV-negative patients.Clin Infect Dis.1997; 24:131-134.

[14] Van der Horst CM,Saag MS,Cloud GA,et al.Treatment of cryptococcal meningitis associated with acquired immunodeficiency syndrome Engl Med.1997;337:15-21.

[15] Bennett JE,Dismukes WE,Duma RJ,et al.A comparison of amphotericin B alone and combined with flucytosine in the treatment of cryptococcal meningitis.N Engl JMed.1979;301:126-131.

[16] Perfect JR.Cryptococcus neoformans:A sugar-coated killer with designer genes. FEMS Immunol Med Microbiol.2005;45:395-404

[17] Steenbergen JN,Shuman HA,Casadevell A.Cryptococcus neoformans interactions with amoebae suggest an explanation for its virulence and intracellular pathogenic strategy in macrophages. Proc Natl Arcad Sci.2001;98:15245-50.

[18] Chang YC,Kwon-Chung KI.Complementation of a capsule deficiency mutation of Cryptococcus neoformans restores its virulence. Moll Cell Biol.1994;14:4912-19.

[19] Casadevall A,Perfect JR.Cryptococcus neoformans.Washington:ASM Press: 1998: 409.

[20] Langfelder K,Streubel N,John R,et al,Biosynthesis of fungal melanins and their importance for human pathogenic fungi..Fungol Genet Biol.2003;38:143-48.

[21] Salas SD,Bennet Je,Kwon-Chung KI,et al.Effect of lactose gene.CNLACI,on virulence of Cryptococcus neoformans.J Exp Med.1996;184:377-86.

[22] Norsanchuk,JD,Rosas AI,Lee SC.Melanization of Cryptococcus neoformans in human brain tissue.Lancet.2000;355:2049-50.

[23] Martinez IR,Garcia-Rivera J,Casadevell A.Cryptococcus neoformans var.nerformans (serotypeD) strains are more susceptible to heat than C.neoformans vargrubii(serotype A strain).J Clin Microbiol.2001;39:3365-67.

[24] Odom A,Muit S,Lim E, et al.Calcineurin is required for virulence of Cryptococcus neoformans .EMBO J.1997;16:2576-89.

[25] Fraser JA,Giles SS,Wenink EC,et al.Same-sex mating and the origin of the Vancouver Island Cryptococcus gatti outbreak.Nature.2005;437:1369-64.

[26] Levitz SM,Overview of host defenses in fungal infections.Clin Infect Dis. 1992;14:S37-S42

[27] Hill J0.CD4-T cells cause multinucleated cells to form around Cryptococcus neoformans and confine the yeast within the primary site of infection in the respiratory tract Exp Med.1992; 175:1685-95.

[28] Levitz SM.Macrophage-Cryptococcusinteractions.In:Zwilling RS,Eisentein TK ,ed. Macrophage-Pathogen Interactions.New York:Marcel Dekker:1994;533-43.

[29] Crowe SM,Carlin JB,Stewart KI,et al.Predictive value of CD4 lymphocyte numbers for the development of opportunistic infections and malignancies in HIV-infected patients Acquir Immune Defic Syndr.1991;4:770-76

[30] Vecchiarelli A,Casadevall A.Antibody- mediated effects against Cryptococcus neoformans.Evidence for interdependency and collaboration between humoral and cellular immunity.Res Immunol.1998;149:321-33.

[31] Larsen Ra,Pappas PG,Perfect JR,et al. Phase I evaluation of the safety and pharmacokinetics of murine-derived anticryptococcal antibody 1887 in subjects with treated cryptococcal meninigitis.Antimicrob Agents Chemother.2005;49:952-58.

[32] Perfect JR.Cryptococcus neoformans.In:Mandell,Douglas and Bennett'sPriciples andPractice of Infectious Diseases, $7^{\text {th }}$ ed,Mandell GI,Bennett JE, Dolin R(editors). Churchill Livingstone Elsevier,2010.

[33] Baker RD.The primary lymph node complex of Cryptococcus.Am J Pathol. 1976;65:83-92.

[34] Garcia-Hemoso D,Johson G,Dromer F.Epidemiological evidence of dormant Cryptococcus neoformans infection.J Clin Microbiol.1999;37:3204-09.

[35] Perfect JR.Cryptococcosis.Infect Dis North Am.1989;3:77-102.

[36] Pappas PG,Perfect JR,Clond GA,et al.Cryptococcosis in HIV negative patients in the era of effective azole therapy.Clin Infect Dis.2001;33:690-99. 
[37] Clark RA,Greer D,Atkinson W,et al.Spectrum of Cryptococcus neoformans infection in 68 patients infected with acquired immunodeficiency virus.Rev Infect Dis. 1990;12:768-77.

[38] Woods MI,MacGinley R,Eisen DP,et al.HIV combination therapy:Partial immune restitution unmasking latent cryptococcal infection.AIDS.1998:12:1491-94.

[39] Jenny -Avital ER,Abadi M.Immune reconstitution cryptococcosis after initiation of successful highly active antiviral therapy.Infect Immun.2002;35:128-133.

[40] Cinti SK,Armstrong WS,Kaufman CA.Recurrence of increased intracranial pressure with antiviral therapy in AIDS patients with cryptococcal meningitis.Mycoses.2003;44:497-501.

[41] Mitchel DH,Sorrell TC.Allworth AM,et al.Cryptococcal disease of CNS in immunocompetent hosts:influence of cryptococcal varierty in clinical manifestations and outcome. Clin Infect Dis.1995;20:611-616.

[42] Goodman JS,Kaufman J,Loening MG.Diagnosis of cryptococcal meningitis:Detection of cryptococcal antigen. $N$ Engl $J$ Med.1971;285:434-436.

[43] Kaufman CA,Bergman Ag,Severance PJ,et al.Detectiion of cryptococcal antigen,Comparison of two latex agglutination tests.Am J Clin Pathol.1981;75:106-109.

[44] Snow RM,Dismukee WE.Cryptococcal meningitis:Diagnostic value of cryptococcal antigen in cerebrospinal fluid..Arch Intern Med.1975;135:1155-57.

[45] El-Zaafri M,PasarellJ,MacGinnis MR,et al.Evaluation of the up-dated Vitek yeast identification data base.J Clin Microbiol.1990;28:1938-41.

[46] Hufllingale KE,Gander RM,Evaluation of Gen-probe's Histoplasma capsulatum and Cryptococcus neoformans ACCU probes.J Clin Microbiol.1993;31:419-421.

[47] Staib F,Cryptococcus neoformans and Guizoria abyssinica (syn.G.aleifera) Farbreaktion for C.neoformans.Zbl Hyg.1962;148:466475.

[48] Sarosi GA,Parker JD,Doto IL,et al.Amphotericin B in Cryptococcus meningitis:Long term results of treatment.Am Intern Med.1969;71:1079-1087.

[49] Bennett JE,Dismukes W,Duma RJ,et al.Comparison of amphotericinn B and combined with flucytosine in the treatment of cryptococcal meningitis.N Engl J Med.1979;501:126-131.

[50] del.alla F,Pellizer G,Vaglia A.Amphotericin B as primary therapy for cryptococcosis in patients AIDS: Reliability of relatively high doses administered over a relatively short period.Clin Infect Dis.1995;20:263-266.

[51] Leenders AC,Reiss P,Portegies P,et al.Liposomal amphotericin B(AmBisome) compared with amphotericin B both followed by oral flucocytosine in the treatment of AIDS-associated cryptococcal meningitis.AIDS, 1997;11:1463-71

[52] Utz JP,Shadomy S,McGehee RF.Flucocytosine.N Engl J Med.1972;286:777-778. 